\title{
Management of chronic wounds in patients with dystrophic epidermolysis bullosa: challenges and solutions
}

\author{
Ellie Rashidghamat ${ }^{\prime}$ \\ Jemima E Mellerio ${ }^{1,2}$ \\ 'St John's Institute of Dermatology, \\ King's College London, ${ }^{2} \mathrm{St}$ John's \\ Institute of Dermatology, Guy's and \\ St Thomas' NHS Foundation Trust, \\ London, UK
}

This article was published in the following Dove Press journal:

Chronic Wound Care Management and Research

14 February 2017

Number of times this article has been viewed

\begin{abstract}
Epidermolysis bullosa (EB) is a clinically and genetically heterogeneous group of severe inherited blistering diseases that affects 500,000 individuals worldwide. Clinically, individuals with EB have fragile skin and are susceptible to blistering following minimal trauma and show involvement of mucus membrane and other organs in some subtypes. Dystrophic EB (DEB) is divided into 2 major types depending on the inheritance pattern: recessive DEB (RDEB) and dominant DEB (DDEB). RDEB tends to be at the more severe end of the clinical spectrum and has a prevalence of 8 per 1 million of the population, accounting for approximately $5 \%$ of all cases of EB. RDEB is caused by loss-of-function mutations in the type VII collagen gene, COL7A1, which leads to reduced or absent type VII collagen (C7) and structurally defective anchoring fibrils at the dermal-epidermal junction. In this review, we will discuss the management of chronic wounds in individuals with DEB, highlighting the changes to practice and the novel therapies that may offer a solution to this debilitating and complex problem which is one of the greatest sources of morbidity in this disease.
\end{abstract}

Keywords: epidermolysis bullosa, recessive dystrophic, dominant dystrophic, wound healing

\section{Introduction}

Epidermolysis bullosa (EB) encompasses a group of genetic conditions with the unifying characteristic feature being the presence of recurrent blistering and erosions, caused by minor mechanical trauma, affecting the skin and certain epithelial-lined tissues. ${ }^{1}$ Dystrophic EB (DEB) is one of the major forms of EB and can be inherited in an autosomal recessive or dominant pattern. The recessive DEB (RDEB) variants tend to be more severe clinically. The prevalence of RDEB has been estimated to be 8 per 1 million of the population ${ }^{2}$ and makes up approximately $5 \%$ of all cases of EB. RDEB is caused by loss-of-function mutations in the type VII collagen gene, COL7A1, which lead to reduced or absent type VII collagen (C7) and structurally defective anchoring fibrils at the dermal-epidermal junction (DEJ). ${ }^{3}$ No proven cure has been established for any form of EB and current management strategies are aimed at providing symptomatic relief, for example, management of pain and itch, treatment of problems such as chronic wounds and infection, and prevention of complications, where possible. ${ }^{4,5}$ In the more severe forms of EB, including RDEB, extensive areas of denuded skin pose a risk of skin infection; therefore, prevention and management of bioburden are important, as wounds that are chronically colonized heal poorly and slowly leading to chronic wound formation. In some EB subtypes, notably the generalized severe form of RDEB, chronic wounds may promote the development of aggressive squamous cell carcinoma which is the leading cause of death in early adulthood. ${ }^{6}$ Managing chronic
Correspondence: Jemima E Mellerio St John's Institute of Dermatology, 3rd Floor, Block C, South Wing, St Thomas Hospital, London SEI 7EH, UK

Tel +44207188 6399

Fax +44207I88 6379

Email jemima.mellerio@kcl.ac.uk 
wounds to promote healing may have an important role, therefore, in reducing the risk of this devastating complication of RDEB.

In this review, we will discuss the current principles of managing chronic wounds in DEB as well as the novel, emerging therapies that may address this difficult and complex area in the future.

\section{Cutaneous wound healing}

Normal cutaneous healing is a complex restorative physiological process in response to tissue injury. Acute wound healing can be subdivided into 4 dynamic and overlapping phases: hemostasis, inflammation, proliferation, and remodeling/maturation. ${ }^{7}$ Following tissue injury, extravasation of blood from the damaged vessels and outflow of lymphatic fluid activate the intrinsic and extrinsic clotting cascades, resulting in fibrin clot formation. This clot induces hemostasis and serves as a matrix for the inflammatory cells.

Within 6-8 hours, the inflammatory phase of healing commences. Platelets degranulate releasing alpha granules, which secrete the growth factors (GFs) such as epidermal growth factor (EGF), platelet-derived growth factor (PDGF), and transforming growth factor beta $(\mathrm{TGF}-\beta) .{ }^{8}$ TGF- $\beta$ released from the injured cells and platelets facilitates the migration of polymorphonuclear leukocytes from the surrounding blood vessels. Polymorphonuclear leukocytes attain their maximum numbers within 24-48 hours. Monocytes become macrophages upon exit from the blood vessels and orchestrate the multiplication of endothelial cells. Factors released from macrophages that influence wound healing include TGFs, cytokines and interleukin-1 (IL-1), tumor necrosis factor (TNF), and PDGF. The proinflammatory cytokine IL-1 attracts neutrophils to the wound, with phagocytosis of bacteria and tissue debris. Neutrophil infiltration usually ceases within a few days of injury. The length of the inflammatory phase depends on the extent of the wound, contamination or infection after injury, and host immune factors.

In the third phase of wound healing, fibroblasts and endothelial cells proliferate under the influence of vascular endothelial GF and fibroblast GF, with the formation of new blood vessels (angiogenesis). Initially, excess collagen type III deposition is seen, with the fibroblasts then migrating into the wound, laying down collagen types I and III. Fibroblasts are the principal source of extracellular matrix (ECM) constituents, serving as a scaffold for the cells and cytoskeletal elements that facilitate contraction of healing wounds. Fibroblasts biochemically alter the ECM by degrading fibrin and producing collagen. ${ }^{9}$

During the remodeling phase, that can last for months or years following the initial injury, the ECM undergoes continuous synthesis and remodeling modulated by direct cell-cell and cell--matrix interactions and under the influence of a number of matrix metalloproteinases (MMPs), reaching a steady state 21 days after the tissue injury. Keratinocytes then migrate and proliferate at the wound margin along the new ECM, enabling re-epithelialization and granulation tissue formation. ${ }^{10}$ Remodeling is achieved by specific MMPs influenced by TGF- $\beta$, PDGF, IL- 1 , and EGF. Fibroblasts migrate into the extracellular wound matrix, differentiating into myofibroblasts. These myofibroblasts synthesize and deposit ECM components that replace the provisional matrix. Myofibroblasts resemble contractile smooth muscle cells and facilitate wound contraction and closure through the expression of alpha-smooth muscle actin in microfilament bundles. ${ }^{11}$

\section{Chronic wounds}

Nonhealing wounds fail to progress through the normal sequential process of wound repair. When these phases are disrupted, prolonged, or incomplete, wounds remain in a chronic and inflammatory state. Chronic wounds can be difficult to treat due to the increased presence of inflammatory cells that degrade the ECM and GFs that are required for wound healing to occur. ${ }^{12} \mathrm{~A}$ number of factors are known to contribute to chronic wounds, including systemic comorbidities such as diabetes mellitus and malnutrition, and local stimuli such as infection, foreign bodies, tissue hypoxia, and trauma, all of which are capable of sustaining a continued influx of neutrophils and macrophages, thereby attenuating the wound response.

\section{EB chronic wounds}

Wound care has been highlighted as a priority and fundamental issue for patients, carers, and health care professionals. ${ }^{13}$ In 2012, the average annual cost of EB in the UK was $€ 19,758$ per patient. ${ }^{14}$ Daily wound care costs in RDEB have been calculated in the pediatric population to range from $\$ 10.43$ for the least expensive dressings options to $\$ 127.54$ for the most expensive options in a neonate boy. Wound care costs ranged from $\$ 22.15$ to $\$ 270.92$ daily for an infant boy and from $\$ 54.54$ to $\$ 668.23$ for a 10 -year-old boy. ${ }^{15}$ Data from the UK suggest that dressing costs in adults with RDEB may exceed $£ 500,000$ per annum, and that costs of having paid carers to undertake dressing changes may also exceed 
$£ 50,000$ each year (JE Mellerio, personal communication, St John's Institute of Dermatology, 2016).

In EB specifically, intact blisters and open wounds occur at various stages of healing and can be located anywhere on the body, but tend to be more pronounced on trauma-prone areas such as knees, feet, hands, and elbows, where the skin overlies bony prominences (Figures 1 and 2). Wounds may heal with scarring leading to contractures, most notably on the hands and feet, although large joints may also be affected. The changes affecting the hands include flexion contractures of the interphalangeal joints, metacarpophalangeal, and wrist joints, as well as fusion of the digits to each other laterally. Scarring and contractures lead to loss of functional digits and a "mitten" deformity, a term which describes the epidermal

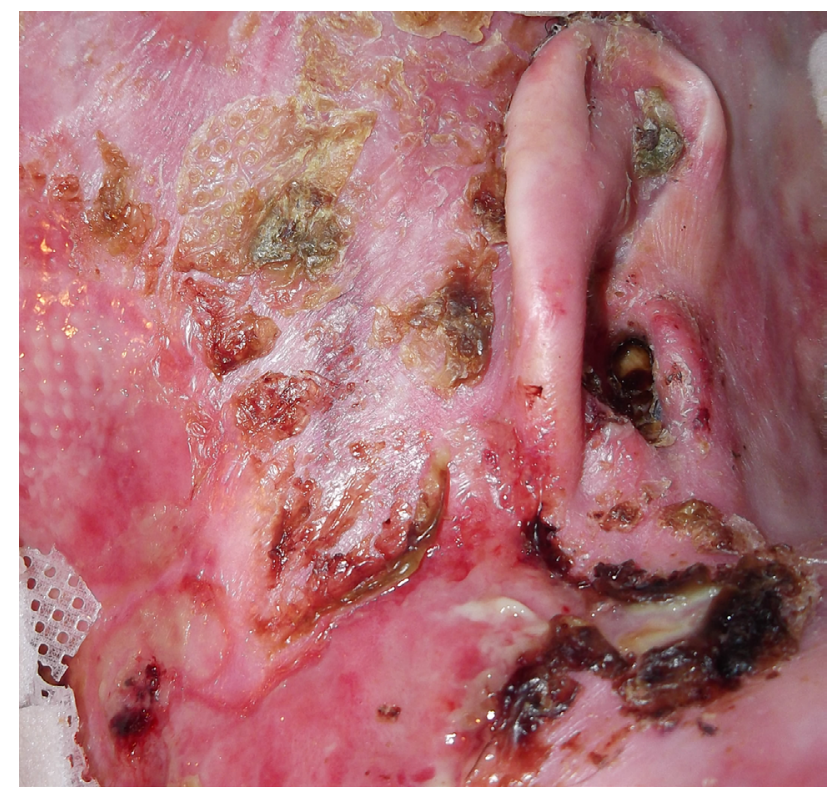

Figure I The right ear and posterior scalp of an individual with RDEB showing the various stages of wounds with erythema, exudate, significant crusting, eschar formation, and scarring.

Abbreviation: RDEB, recessive dystrophic epidermolysis bullosa.

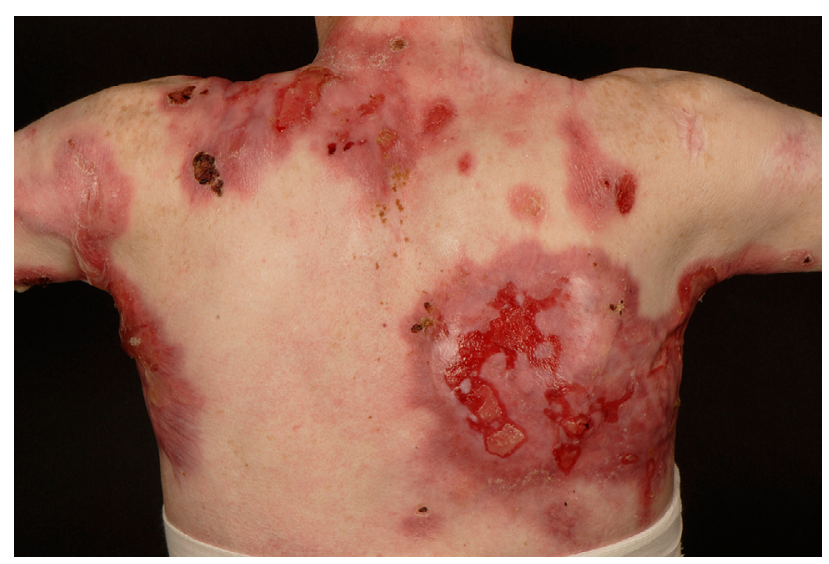

Figure 2 The back of an RDEB patient with fresh erosions, exudate, crusting, eschar formation, and scarring.

Abbreviation: RDEB, recessive dystrophic epidermolysis bullosa. cocooning that encases the hand. ${ }^{16}$ This leads to significant pain for patients when they attempt to extend the affected joints ${ }^{17,18}$ as well as significant functional impairment. Colonization, critical colonization, and recurrent infections of wounds are also a common problem because of extensive areas of denuded skin and the accumulation of serum and moisture on the surface, which enhances the growth of bacteria. ${ }^{19}$

Pruritus is a common problem in all types of EB, particularly in the more severe forms of DEB and in those with a greater body surface area involvement. ${ }^{20}$ Scratching of the fragile skin in EB can lead to very significant areas of eroded skin and exacerbates the tendency to develop chronic wounds. Although the primary cause of pruritus in EB remains unclear, it has been postulated that dry skin and increased heat and moisture from dressings contribute to the intensity of itch and that wound healing and inflammation may contribute to an itch-scratch-blister cycle leading to further skin damage. ${ }^{21}$ Therefore, moisturizing the skin with emollients may reduce the itching tendency. ${ }^{22,23}$

A number of factors in EB make wound healing potentially more complicated, such as anemia and malnutrition. Most individuals with severe generalized RDEB have a mixed picture of iron deficiency anemia due to blood loss from the wounds, as well as anemia of chronic disease due, in large measure, to inflammation from chronic wounds. They are often refractory to oral iron supplements, ${ }^{24}$ necessitating intravenous iron infusions or blood transfusions. Dietary intake is often restricted due to oral scarring, dental caries, and esophageal strictures, and nutritional requirements are greater in severe EB due to insensible losses and thermal dysregulation from chronic wounds contributing to a hypercatabolic inflammatory state. ${ }^{25}$ As a result, the severity of EB often correlates with the degree of energy and protein malnutrition that can impact negatively on the wound healing potential.

\section{Standard wound care}

In DEB, management of multiple wounds of varying duration and healing stage is complex. Preventive measures to reduce trauma, such as minimizing any source of friction or padding, are advised. Blisters that do occur following trauma and minor friction extend rapidly if left intact, and therefore should be lanced to prevent extension of the blister and further skin damage. ${ }^{26}$ Choice of dressings in EB varies from individual to individual and also depends on the location and characteristics of individual wounds. An ideal dressing in EB should provide the correct moisture balance for healing, 
stay in place on the skin without moving or rucking up yet be atraumatic on removal, conform to difficult anatomic sites such as the neck and axillae, and be available in appropriate sizes to cover potentially large areas of wounds where necessary. ${ }^{23}$ Semi-occlusive dressings that are nonadhesive, such as soft silicone and foam dressings, are preferable as they absorb the exudate and minimize pain and further blister formation during dressing changes. ${ }^{27}$

An important area to address in DEB wound management is wound colonization, critical colonization, and infection. In critically colonized wounds, the bacterial load can be reduced with topical agents such as diluted bleach baths, topical antiseptics, and topical antibiotics. ${ }^{22}$ Wounds showing clinical evidence of frank infection require administration of systemic antibiotics with the choice based on culture results. Moisturizers that contain an antimicrobial agent such as benzalkonium chloride or chlorhexidine dihydrochloride, both found in Dermol ${ }^{\mathrm{TM}}$ products (Dermal Laboratories), can be helpful to reduce bacterial colonization. The exudate can be copious and needs careful containment to avoid maceration of the surrounding skin and leakage. The use of barrier preparations such as Cavilon (3M) or Proshield Plus cream (H \& R Healthcare) can minimize the problem of maceration and secondary skin break down. In the neonatal period and infancy, special attention should be given to emollients used, as some formulations may contain potentially irritating chemicals such as sodium lauryl sulfate that need to be avoided.

For dry to lightly exuding chronic EB wounds, nonadhesive soft silicone or lipido-colloid contact layers are the most appropriate primary dressings to be used when the skin is very fragile, such as Mepitel ${ }^{\circledR}$ (Mölnlycke Healthcare), Adaptic $^{\circledR}$ touch (Systagenix), Urgotul ${ }^{\circledR}$ (Urgo Medical), or Silflex ${ }^{\circledR}$ (Advancis Medical). A soft silicone secondary dressing can be used over this, or directly onto the skin of less fragile patients, for example, Mepilex Lite or Mepilex Transfer (Mölnlycke Healthcare), to absorb light levels of exudate and provide a degree of protection of the wound. Hydrogel dressings can also be applied directly to the skin, for example, Intrasite ${ }^{\circledR}$ Conformable (Smith \& Nephew), Kerralite Cool (Crawford Healthcare), or Actiform Cool (Activa Healthcare). These dressings need to be changed daily or when they become dry, whereas other dressings can be changed every 3-4 days if the exudate levels are manageable. ${ }^{28}$ In more heavily exuding wounds, hydrofiber dressings such as Aquacel ${ }^{\circledR}$ (Convatec) or a soft silicone foam with super-absorbers such as Cutimed ${ }^{\circledR}$ Siltec (BSNmedical) may be helpful. Should the wounds become infected, the same dressings used for heavily exuding wounds can be employed, but should be changed on a daily basis. PolyMem (Ferris) is a polymeric membrane dressing that can absorb significant amounts of exudate and releases a surfactant to cleanse the wounds when colonized or infected, which can be helpful in the EB setting. Silver-containing dressings such as Mepilex Ag (Mölnlycke Healthcare), PolyMem Silver (Ferris), and Urgotul SSD (Urgo Medical) have also been used for limited periods of time for infected EB wounds. All dressings for all types of EB wounds should be checked daily and modified depending on the amount of exudate and odor.

If the wound is covered in an eschar, this can act as a proinflammatory stimulus preventing normal wound healing. Debridement encourages healing by removing the biofilms that sustain the inflammatory process. Care must be taken with debridement in EB patients due to skin fragility, and nonphysical methods such as hydrogel or calcium alginate dressings are preferential to physical methods of debridement. ${ }^{22}$ Topical application of medical grade honey has been shown to facilitate debridement which may be useful to promote healing in EB wounds, ${ }^{29}$ although stinging and increased exudate levels may limit its tolerability. Another potential topical treatment for chronic EB wounds is a keratin gel which has been shown to increase the proliferation and migration of keratinocytes with increased collagen 4 and collagen 7 deposition in venous and diabetic ulcers. ${ }^{30} \mathrm{~A}$ small case series in patients with various types of EB suggested that some derived benefit from its use. ${ }^{31}$

\section{Drug therapy}

Various systemic drugs have been trialed to ascertain whether they lead to less blistering and, therefore, cause an improvement in the clinical phenotype. Trimethoprim is an antibiotic that was assessed for its anti-inflammatory effect in a pilot double-blind, randomized, placebo-controlled, crossover trial. Although the study was not adequately powered to achieve statistical significance, the trend showed improved wound healing with trimethoprim compared to placebo, ${ }^{32}$ although further work is required to show its clinical benefit to patients in larger trials.

Tetracyclines are broad-spectrum anti-inflammatory antibiotics and have been the subject of a clinical trial in EB simplex patients, although the small sample size meant that despite showing a trend for reduced blistering, significance was not reached. ${ }^{33}$ Minocycline has also been used in DEB on the basis that tetracyclines (including minocycline) have anti-collagenase activity. ${ }^{34}$ This led to a reduction in blistering, ${ }^{35}$ which was thought to be secondary to a reduction in 
MMP $-9^{36}$ that is usually raised in DEB. ${ }^{37}$ However, large clinical trials have not been conducted to evaluate whether it can be recommended in clinical practice, and concerns exist over its long-term use in view of the risks of side effects such as skin pigmentation and drug-induced connective tissue disease. Phenytoin is an anticonvulsant that also inhibits collagenase activity and had been trialed to assess its benefit in RDEB blistering ${ }^{38}$ before a multicenter randomized, placebo-controlled, double-blind, crossover study showed it had no significant therapeutic effect. ${ }^{39}$

A role for immune suppressants such as ciclosporin and mycophenolate mofetil has been explored, although trial design without appropriate controls makes the interpretation difficult. ${ }^{40}$ Furthermore, given the propensity for development of aggressive squamous cell carcinomas in the RDEB population, routine use of such immunosuppressant agents would be hard to justify. In a single case report, the TNF- $\alpha$ inhibitor etanercept showed some clinical improvement in pruritus and blistering in DEB, when given for coexisting psoriatic arthritis. $^{41}$

Thalidomide is an immunomodulatory drug that has been used in EB pruriginosa because of its established use in nodular prurigo. ${ }^{42}$ It has been reported to be safe and improves pruritus. There is also some evidence that it may increase keratinocyte migration and proliferation, leading to wound healing. ${ }^{43,44}$

Serendipitously, it was observed that a patient with RDEB undergoing bone marrow transplantation (BMT; Table 1) for her disease had a significant improvement in wound healing during autologous peripheral blood stem cell mobilization with systemic granulocyte colony-stimulating factor before the transplant. On the back of this, a pilot trial showed that granulocyte colony-stimulating factor given as 6 daily doses (10 $\mu \mathrm{g} / \mathrm{kg} / \mathrm{dose}$ ) to patients with DEB (6 RDEB, 1 dominant DEB) was safe. In addition, patients had a mean reduction of $75.5 \%$ in lesion size and $36.6 \%$ in blister/erosion counts. ${ }^{45}$

Animal studies have evaluated whether thymosin $\beta 4$, a small molecular weight protein that promotes the migration and adherence of keratinocytes and the upregulation of laminin-332, enhances wound healing when applied topically. ${ }^{46}$ However, completion of clinical trials in EB patients has not yet been achieved to evaluate its use in this setting.

\section{Cellular and acellular matrix products}

When standard wound care fails, cellular and acellular matrix products can be used to treat chronic wounds. Cellular matrices provide cells, GFs, and other key elements that promote re-epithelialization and revascularization of the wound bed, while preventing the degradation of ECM. Acellular matrices can be biologically active or biologically inert and act as a scaffold for cellular migration and endogenous matrix production. An advantage of acellular matrices is that they have less potential to cause an immunogenic reaction.

In a study, a cultured epidermal autograft was manufactured by taking a full-thickness biopsy specimen of the skin from an

Table I Summary of the published clinical studies using advanced therapeutic approaches for wound management in RDEB

\begin{tabular}{|c|c|c|c|}
\hline Advanced therapy & Study type & Outcome & References \\
\hline Cultured dermal substitute & $\begin{array}{l}\text { Prospective interventional } \\
\text { study }\end{array}$ & $\begin{array}{l}\text { Granulation found on wound surface with } \\
\text { epithelialization }\end{array}$ & Hasegawa et $\mathrm{al}^{48}$, Natsuga et $\mathrm{al}^{49}$ \\
\hline Amniotic membrane grafting & $\begin{array}{l}\text { Retrospective, } \\
\text { proof-of-concept study }\end{array}$ & $\begin{array}{l}\text { Promoted healing of nonhealing wounds in } \\
\text { EB with pain reduction, but not complete } \\
\text { re-epithelialization }\end{array}$ & Lo et $\mathrm{al}^{54}$ \\
\hline Fibroblasts & Proof-of-concept study & $\begin{array}{l}\text { Increased } C 7 \text { observed, particularly in patients } \\
\text { with some baseline } C 7\end{array}$ & Wong et $\mathrm{al}^{55}$ \\
\hline Fibroblasts & Pilot study in a single subject & $\begin{array}{l}\text { Increased COL7AI expression for } 3-6 \text { months and } \\
\text { C7 protein for } 9-12 \text { months }\end{array}$ & Nagy et $\mathrm{al}^{56}$ \\
\hline Fibroblasts & $\begin{array}{l}\text { Prospective, randomized, } \\
\text { double-blind, within-patient, } \\
\text { vehicle-controlled trial }\end{array}$ & $\begin{array}{l}\text { Greater reduction in erosion area with fibroblasts } \\
\text { versus control }\end{array}$ & Petrof et $\mathrm{al}^{57}$ \\
\hline MSCs & $\begin{array}{l}\text { Pilot study in } 2 \text { RDEB } \\
\text { subjects }\end{array}$ & $\begin{array}{l}\text { Wounds injected with MSCs almost healed at } \\
\text { week I } 2 \text { compared to placebo }\end{array}$ & Conget et $\mathrm{al}^{59}$ \\
\hline MSCs & Randomized-controlled trial & $\begin{array}{l}\text { Increased rate of wound healing compared to } \\
\text { baseline }\end{array}$ & El-Darouti et $\mathrm{al}^{60}$ \\
\hline MSCs & Open-label, Phase I/II trial & Reduced skin redness and better wound healing & Petrof et $\mathrm{al}^{61}$ \\
\hline Bone marrow transplantation & Open-label intervention trial & $\begin{array}{l}\text { Improved wound healing and reduction in } \\
\text { blister formation between } 30 \text { and } 130 \text { days after } \\
\text { transplantation }\end{array}$ & Wagner et $a^{62}$ \\
\hline
\end{tabular}

Abbreviations: EB, epidermolysis bullosa; MSCs, mesenchymal stromal cells; RDEB, recessive dystrophic epidermolysis bullosa. 
RDEB patient and culturing the keratinocytes to confluence. The cultured epidermal autograft was then grafted onto a chronic wound and epithelialization was observed 2 weeks later, with no evidence of tumorigenesis 10 years on. ${ }^{47}$ Allogeneic cultured dermal substitutes have been used to treat chronic wounds in RDEB with reported success (Table 1). ${ }^{48,49}$ Apligraf $^{\circledR}$ (Organogenesis, Canton, MA, USA) is an allogeneic cultured skin substitute consisting of keratinocytes and fibroblasts supported on a scaffold. Initially, Apligraf was used to treat venous ulcers, but has also been used to treat wounds in EB with some benefit in wound healing reported. ${ }^{50,51}$ Cryopreserved placental membrane (CPM; Grafix, Osiris Therapeutics, Inc., Columbia, MD, USA) is a cellular matrix composed of placental membrane. It is a human viable wound matrix that provides the wound with mesenchymal stem cells, neonatal fibroblasts, epithelial cells, GFs, and angiogenic factors. ${ }^{52}$ One of the licensed uses for Grafix is in the management of acute and chronic wounds in EB. In a randomized-controlled trial, CPM was compared with standard wound care to treat diabetic foot ulcers, wherein significantly higher wound closure was found in the CPM $\operatorname{arm}\left(62 \%\right.$ vs $21 \%$, respectively).$^{53}$

Amniotic membrane has biological properties that can promote wound healing and has been used in EB to promote the healing of chronic wounds and found to be efficacious causing reduction in pain, but without complete re-epithelialization (Table 1 ). ${ }^{54}$

A Phase III interventional clinical trial is currently underway comparing the efficacy of amniotic membrane in healing chronic ulcers in RDEB to standard dressings (ClinicalTrials. gov identifier: NCT02286427).

\section{Cell therapy - fibroblasts, mesenchymal stromal cells (MSCs), and BMT}

Type VII collagen is synthesized by both keratinocytes and fibroblasts. So, to exploit this, allogeneic normal human fibroblasts were injected into patients with severe generalized RDEB to increase C7 expression (Table 1). ${ }^{55}$ An increase in C7 was most apparent in patients who had some baseline C7 present rather than a complete absence. Interestingly, basal keratinocytes showed increased intracellular C7, suggesting that the fibroblast injections had stimulated the production of the patient's own keratinocyte-derived mutant $\mathrm{C} 7$ through a paracrine effect, rather than direct production of $\mathrm{C} 7$ from the injected fibroblasts, which did not persist in the skin. Despite the increased expression of C7, this study did not find evidence of an improvement in anchoring fibril number or morphology. A subsequent study showed that a single injection of allogeneic fibroblasts could increase
COL7A1 expression for 3-6 months and C7 protein for 9-12 months; this was thought to be mediated through an increase in heparin-binding-EGF-like growth factor (HB-EGF) expression. ${ }^{56}$ In addition, a prospective, randomized, doubleblind, within-patient, vehicle-controlled trial of subjects with RDEB showed a trend toward greater reduction in erosion area with fibroblasts than with vehicle alone up to day $28 .{ }^{57}$

Bone marrow-derived MSCs (Table 1) are multipotent stem cells that can be found in the bone marrow and are of nonhematopoietic lineage able to differentiate into a number of different cell types of stromal lineage including keratinocytes and fibroblasts and have been the subject of interest in $\mathrm{EB}$ over recent years. ${ }^{58}$

In an initial study, MSCs from unrelated individuals were injected intradermally into chronic wounds of 2 RDEB patients and compared to placebo injections. ${ }^{59}$ The wound areas injected with MSCs, in contrast to the placebo-treated areas, had almost healed at week 12 and this benefit lasted for 4 months. This pilot study also showed that intradermal administration of allogeneic MSCs led to de novo cutaneous $\mathrm{C} 7$ expression in these patients. In another double-blind study, 14 patients with RDEB were randomized into 2 equal groups. ${ }^{60}$ Both received intravenous MSCs derived from the bone marrow of a healthy parent, but 1 group received ciclosporin and the other a placebo. Both groups showed an increased rate of wound healing compared to baseline. Intravenous infusions of allogeneic MSCs were also administered to children in an open-label, Phase I/II clinical trial ${ }^{61}$ and it showed clinical benefits including reduced skin redness in $10 / 10$ patients, better wound healing $(9 / 10)$, less skin pain $(5 / 10)$, and less skin itching (5/10). However, these changes were not accompanied by increased $\mathrm{C} 7$ deposition at the DEJ, anchoring fibril formation nor donor cell chimerism. However, the use of systemic MSC therapy may represent a useful therapy for RDEB patients, although further studies, some of which are currently underway (ClinicalTrials.gov identifier: NCT02323789), are needed to elucidate the precise mechanisms through which they deliver clinical benefit.

Two trials have been conducted to evaluate the use of BMT in RDEB patients. This approach requires ablation of the bone marrow of the patient and then BMT from an HLAmatched healthy donor with the hope that these multilineage cells can differentiate and improve the clinical phenotype. In 2010, tissue-matched, unfiltered, whole BMT was first reported in patients with $\mathrm{RDEB} .{ }^{62}$ Seven patients entered the trial and 6 underwent BMT as 1 died from complications before transplantation. Following BMT, 3 subjects showed clinical improvement with only $10 \%$ BSA involvement and 3 showed an improvement with 25\% BSA involvement. C7 
expression was seen at the DEJ in 5 of the 6 subjects which persisted over many months with evidence of donor cell chimerism. A further patient died 6 months posttransplant from infection secondary to graft failure, demonstrating the high risk of mortality associated with this intervention. To overcome the toxicity associated with myeloablation, nonmyeloablative trials with reduced intensity conditioning are underway. ${ }^{63}$

A Phase II, single-center trial is determining the 1-year event-free survival post allogeneic transplant and serial mesenchymal stem cell infusions from a related donor (HLA identical, mismatched, or haploidentical) or matched unrelated donor to evaluate whether there is biochemical correction in severe EB (ClinicalTrials.gov identifier: NCT02582775). A local wound therapy trial is also underway using epidermal skin grafts from the same donor that provided the hematopoietic cell transplant to the EB recipient (ClinicalTrials.gov identifier: NCT02670837).

\section{Gene therapy}

Gene addition or augmentation, sequence correction, gene knockdown or exon skipping, as well as gene editing approaches are exciting novel therapeutic approaches aiming to correct the gene defect in RDEB. Through the manipulation of DNA or RNA, it is hoped that there will be a therapeutic benefit to patients. Current gene therapy strategies for EB aim to repair the gene mutations rather than add additional copies of corrected genes using candidate platforms for gene editing including zinc-finger nucleases, meganucleases, transcription activator-like effector nucleases, and the clustered, regularly interspaced, short palindromic repeatsCas9 nuclease system. Splice-modulating technology such as spliceosome-mediated RNA trans-splicing has also been used to excise mutant exons in order to knock out the mutant protein in the affected cells at the posttranscriptional level. Preclinical studies have shown remarkable results in terms of gene correction/excision using both in vivo and ex vivo approaches. ${ }^{64-70}$ In addition, a previous study of ex vivo gene therapy in an individual with junctional EB demonstrated restoration of laminin 332 expression following retroviralmediated transfection of epidermal stem cells with the $L A M B 3$ gene, with long-lasting phenotypic correction in the grafted skin. ${ }^{71}$ A US trial is currently evaluating the safety and efficacy of a retroviral vector and genetically engineered epithelial graft cultured from the skin of patients with RDEB (ClinicalTrials.gov identifier: NCT01263379). A European gene therapy trial (www.genegraft.eu) is using a self-inactivating retroviral vector and ex vivo gene therapy with the aim to transplant bioengineered skin containing genetically modified keratinocytes and fibroblasts. In addition, a UK trial of lentiviral-corrected fibroblasts is also currently underway in RDEB (ClinicalTrials.gov identifier: NCT02493816).

\section{Protein therapy}

Replacement of collagen VII by injecting recombinant human $\mathrm{C} 7$ directly into wounds is an exciting new strand of research in EB that may ameliorate the blistering phenotype resulting from the absence or reduced expression of C7. Preclinical studies $^{72}$ have shown that recombinant human $\mathrm{C} 7$ delivered by intradermal injection is capable of migrating to the basement membrane and being incorporated into the DEJ of Col7al null mice, causing an improvement in the blistering phenotype for up to 2 months. Topical application of human recombinant $\mathrm{C} 7$ (rC7) accelerated wound healing ${ }^{73}$ in mice, and intravenously administered $\mathrm{rC} 7$ homed to engrafted RDEB mouse skin and restored C7, anchoring fibrils, and epidermal-dermal adherence. ${ }^{72,74}$ Further animal studies in inbred golden retriever dogs with mild RDEB revealed that intravenous administration of $\mathrm{rC} 7$ results in reduced wound erythema and blistering. ${ }^{75}$ Mechanistic data are still lacking and there is potential to develop clinically relevant antibodies to $\mathrm{C} 7 .^{76,77}$ This therapy has not yet been translated into a model suitable for clinical application, but remains a hopeful approach for future therapeutic development.

\section{Fractionated $\mathrm{CO}_{2}$ laser for $\mathrm{EB}$ wounds}

Ablative fractional laser resurfacing (AFR) is an emerging therapy for treating chronic wounds. A single case report used a fractionated $\mathrm{CO}_{2}$ laser protocol in a 22-year-old man with RDEB to accelerate wound healing. ${ }^{69}$ The proposed mechanism of action of AFR is postulated to be multifactorial. Photo-microdermabrasion, similar to conventional surgical debridement, may provide benefits without the need for downtime often associated with surgery. AFR may also stimulate collagen stimulation and secretion of GFs, promoting healing. Based on a single case report, it is difficult to determine the true efficacy and further studies are required. ${ }^{78}$

\section{Conclusion}

Although our understanding of the pathophysiology of EB has increased and significant advances have been made toward treating and preventing wounds, given the complexity of EB, it is unlikely that a single treatment will reverse the clinical phenotype or its sequelae. New pharmacotherapeutic approaches are being explored to both treat and prevent some of the downstream effects of EB and have shown rapid advances in 
the arena of regenerative medicine. Several of these advances are being utilized in the clinical setting today. Drug therapies are also being streamlined to target specific pathophysiologic mechanisms. However, the limited number of RDEB individuals makes large-scale clinical trials challenging to confirm the safety and efficacy of novel therapies. Potentially being able to cure this heritable skin disease is becoming conceivable and showing promise that we are getting closer.

\section{Acknowledgment}

Patients provided verbal consent for their images to be used in this review.

\section{Disclosure}

The authors report no conflicts of interest in this work.

\section{References}

1. Bruckner-Tuderman L. Dystrophic epidermolysis bullosa: pathogenesis and clinical features. Dermatol Clin. 2010;28(1):107-114.

2. Fine JD. Inherited epidermolysis bullosa. Orphanet J Rare Dis. 2010;5:12.

3. van den Akker PC, Jonkman MF, Rengaw T, et al. The international dystrophic epidermolysis bullosa patient registry: an online database of dystrophic epidermolysis bullosa patients and their COL7A1 mutations. Hum Mutat. 2011;32(10):1100-1107.

4. Mellerio JE, Weiner M, Denyer JE, Pillay EI, Lucky AW, Bruckner A, Palisson F. Medical management of epidermolysis bullosa: proceedings of the IInd International Symposium on Epidermolysis Bullosa, Santiago, Chile, 2005. Int J Dermatol. 2007;46(8):795-800.

5. Azizkhan RG, Denyer JE, Mellerio JE, et al. Surgical management of epidermolysis bullosa: proceedings of the IInd International Symposium on Epidermolysis Bullosa, Santiago, Chile, 2005. Int J Dermatol. 2007;46(8):801-808.

6. Fine JD, Johnson LB, Weiner M, Li KP, Suchindran C. Epidermolysis bullosa and the risk of life-threatening cancers: the National EB Registry experience, 1986-2006. J Am Acad Dermatol. 2009;60(2):203-211.

7. Clark RA. Wound repair. Curr Opin Cell Biol. 1989;1(5):1000-1008.

8. Barrientos S, Stojadinovic O, Golinko MS, Brem H, Tomic-Canic M. Growth factors and cytokines in wound healing. Wound Repair Regen. 2008;16(5):585-601.

9. Lorenz HP, Longaker MT, Perkocha LA, Jennings RW, Harrison MR, Adzick NS. Scarless wound repair: a human fetal skin model. Development. 1992;114(1):253-259.

10. Gurtner GC, Werner S, Barrandon Y, Longaker MT. Wound repair and regeneration. Nature. 2008;453(7193):314-321.

11. Hinz B, Gabbiani G. Cell-matrix and cell-cell contacts of myofibroblasts: role in connective tissue remodeling. Thromb Haemost. 2003;90(6):993-1002.

12. Sibbald RG, Goodman L, Woo KY, et al. Special considerations in wound bed preparation 2011: an update(c). Adv Skin Wound Care. 2011;24(9):415-436; quiz 437-438.

13. Davila-Seijo P, Hernández-Martín A, Morcillo-Makow E, et al. Prioritization of therapy uncertainties in dystrophic epidermolysis bullosa: where should research direct to? An example of priority setting partnership in very rare disorders. Orphanet J Rare Dis. 2013;8:61.

14. Angelis A, Kanavos P, López-Bastida J, et al. Social/economic costs and health-related quality of life in patients with epidermolysis bullosa in Europe. Eur J Health Econ. 2016;17(Suppl 1):31-42.

15. Kirkorian AY, Weitz NA, Tlougan B, Morel KD. Evaluation of wound care options in patients with recessive dystrophic epidermolysis bullosa: a costly necessity. Pediatr Dermatol. 2014;31(1):33-37.

16. Bernardis C, Box R. Surgery of the hand in recessive dystrophic epidermolysis bullosa. Dermatol Clin. 2010;28(2):335-341, xi.
17. Denyer J. Managing pain in children with epidermolysis bullosa. Nurs Times. 2012;108(29):21-23.

18. Goldschneider KR, Good J, Harrop E, et al. Pain care for patients with epidermolysis bullosa: best care practice guidelines. BMC Med. 2014;12(1): 178 .

19. Pillay E. Epidermolysis bullosa. Part 1: causes, presentation and complications. Br J Nurs. 2008;17(5):292-296.

20. van Scheppingen C, Lettinga AT, Duipmans JC, Maathuis CG, Jonkman MF. Main problems experienced by children with epidermolysis bullosa: a qualitative study with semi-structured interviews. Acta Derm Venereol. 2008;88(2):143-150.

21. Danial C,Adeduntan R, Gorell ES, et al. Prevalence and characterization of pruritus in epidermolysis bullosa. Pediatr Dermatol. 2015;32(1):53-59.

22. Pope E, Lara-Corrales I, Mellerio J, et al. A consensus approach to wound care in epidermolysis bullosa. J Am Acad Dermatol. 2012; 67(5):904-917.

23. Denyer JE. Best Practice Guidelines for Skin and Wound Care in Epidermolysis Bullosa. International Consensus. DEBRA; 2012.

24. Fridge JL, Vichinsky EP. Correction of the anemia of epidermolysis bullosa with intravenous iron and erythropoietin. J Pediatr. 1998; 132(5):871-873.

25. Colomb V, Bourdon-Lannoy E, Lambe C, et al. Nutritional outcome in children with severe generalized recessive dystrophic epidermolysis bullosa: a short- and long-term evaluation of gastrostomy and enteral feeding. Br J Dermatol. 2012;166(2):354-361.

26. Denyer JE. Wound management for children with epidermolysis bullosa. Dermatol Clin. 2010;28(2):257-64, viii-ix.

27. Caldwell-Brown GS, Reid M. Nursing Aspects of Epidermolysis Bullosa: A Comprehensive Approach. Basic and Clinical Aspects: SpringerVerlag; 1992, New York, NY.

28. El Hachem M, Zambruno G, Bourdon-Lanoy E, et al. Multicentre consensus recommendations for skin care in inherited epidermolysis bullosa. Orphanet J Rare Dis. 2014;9:76.

29. Hon J. Using honey to heal a chronic wound in a patient with epidermolysis bullosa. Br J Nurs. 2005;14(19 Suppl):S4-S12.

30. Batzer AT, Marsh C, Kirsner RS. The use of keratin-based wound products on refractory wounds. Int Wound J. 2016;13(1):110-115.

31. Denyer J, Marsh C, Kirsner RS. Keratin gel in the management of epidermolysis bullosa. $J$ Wound Care. 2015;24(10):446-450.

32. Lara-Corrales I, Parkin PC, Stephens D, et al. The efficacy of trimethoprim in wound healing of patients with epidermolysis bullosa: a feasibility trial. J Am Acad Dermatol. 2012;66(2):264-270.

33. Weiner M, Stein A, Cash S, de Leoz J, Fine JD. Tetracycline and epidermolysis bullosa simplex: a double-blind, placebo-controlled, crossover randomized clinical trial. Br J Dermatol. 2004;150(3):613-614.

34. Golub LM, Stein A, Cash S, de Leoz J, Fine JD. Further evidence that tetracyclines inhibit collagenase activity in human crevicular fluid and from other mammalian sources. J Periodontal Res. 1985;20(1):12-23.

35. White JE. Minocycline for dystrophic epidermolysis bullosa. Lancet. 1989;1(8644):966.

36. Leung J, Kuzel P, Kurian A, Brassard A. A case of dominant dystrophic epidermolysis bullosa responding well to an old medication. JAMA Dermatol. 2015;151(11):1264-1265.

37. Lettner T, Lang R, Bauer JW, Wally V. Increased levels of matrix metalloproteinase- 9 and interleukin-8 in blister fluids of dystrophic and junctional epidermolysis bullosa patients. J Eur Acad Dermatol Venereol. 2015;29(2):396-398.

38. Bauer EA, Cooper TW, Tucker DR, Esterly NB. Phenytoin therapy of recessive dystrophic epidermolysis bullosa. Clinical trial and proposed mechanism of action on collagenase. $N$ Engl J Med. 1980; 303(14):776-781.

39. Caldwell-Brown D, Stern RS, Lin AN, Carter DM. Lack of efficacy of phenytoin in recessive dystrophic epidermolysis bullosa. Epidermolysis Bullosa Study Group. N Engl J Med. 1992;327(3):163-167.

40. El-Darouti MA, Fawzy MM, Amin IM, Abdel Hay RM, Hegazy RA, Abdel Halim DM. Mycophenolate mofetil: a novel immunosuppressant in the treatment of dystrophic epidermolysis bullosa, a randomized controlled trial. J Dermatolog Treat. 2013;24(6):422-426. 
41. Gubinelli E, Angelo C, Pacifico V. A case of dystrophic epidermolysis bullosa improved with etanercept for concomitant psoriatic arthritis. Am J Clin Dermatol. 2010;11(Suppl 1):53-54.

42. Wines NY, Cooper AJ, Wines MP. Thalidomide in dermatology. Australas J Dermatol. 2002;43(4):229-238; quiz 239-240.

43. Ozanic Bulic S, Fassihi H, Mellerio JE, McGrath JA, Atherton DJ. Thalidomide in the management of epidermolysis bullosa pruriginosa. Br J Dermatol. 2005;152(6):1332-1334.

44. Ishida Y, Kondo T, Takayasu T, Iwakura Y, Mukaida N. The essential involvement of cross-talk between IFN-gamma and TGF-beta in the skin wound-healing process. J Immunol. 2004;172(3):1848-1855.

45. Fine JD, Manes B, Frangoul H. Systemic granulocyte colony-stimulating factor (G-CSF) enhances wound healing in dystrophic epidermolysis bullosa (DEB): results of a pilot trial. J Am Acad Dermatol. 2015 73(1):56-61.

46. Fine JD. Epidermolysis bullosa: a genetic disease of altered cell adhesion and wound healing, and the possible clinical utility of topically applied thymosin beta4. Ann N Y Acad Sci. 2007;1112:396-406.

47. Shinkuma S, Sawamura D, Fujita Y, et al. Long-term follow-up of cultured epidermal autograft in a patient with recessive dystrophic epidermolysis bullosa. Acta Derm Venereol. 2014;94(1):98-99.

48. Hasegawa T, Suga Y, Mizoguchi M, et al. Clinical trial of allogeneic cultured dermal substitute for the treatment of intractable skin ulcers in 3 patients with recessive dystrophic epidermolysis bullosa. J Am Acad Dermatol. 2004;50(5):803-804.

49. Natsuga K, Sawamura D, Goto M, et al. Response of intractable skin ulcers in recessive dystrophic epidermolysis bullosa patients to an allogeneic cultured dermal substitute. Acta Derm Venereol. 2010;90(2): 165-169.

50. Fivenson DP, Scherschun L, Cohen LV. Apligraf in the treatment of severe mitten deformity associated with recessive dystrophic epidermolysis bullosa. Plast Reconstr Surg. 2003;112(2):584-588.

51. Falabella AF, Valencia IC, Eaglstein WH, Schachner LA. Tissueengineered skin (Apligraf) in the healing of patients with epidermolysis bullosa wounds. Arch Dermatol. 2000;136(10):1225-1230.

52. Gibbons GW. Grafix ${ }^{\circledR}$, a cryopreserved placental membrane, for the treatment of chronic/stalled wounds. Adv Wound Care (New Rochelle). 2015;4(9):534-544.

53. Lavery LA, Fulmer J, Shebetka KA, et al. The efficacy and safety of $\operatorname{Grafix}((\mathrm{R}))$ for the treatment of chronic diabetic foot ulcers: results of a multi-centre, controlled, randomised, blinded, clinical trial. Int Wound J. 2014;11(5):554-560.

54. Lo V, Lara-Corrales I, Stuparich A, Pope E. Amniotic membrane grafting in patients with epidermolysis bullosa with chronic wounds. $J \mathrm{Am}$ Acad Dermatol. 2010;62(6):1038-1044.

55. Wong T, Gammon L, Liu L, et al. Potential of fibroblast cell therapy for recessive dystrophic epidermolysis bullosa. J Invest Dermatol. 2008;128(9):2179-2189.

56. Nagy N, Almaani N, Tanaka A, Lai-Cheong JE, Techanukul T, Mellerio JE, McGrath JA. HB-EGF induces COL7A1 expression in keratinocytes and fibroblasts: possible mechanism underlying allogeneic fibroblast therapy in recessive dystrophic epidermolysis bullosa. J Invest Dermatol. 2011;131(8):1771-1774

57. Petrof G, Martinez-Queipo M, Mellerio JE, Kemp P, McGrath JA. Fibroblast cell therapy enhances initial healing in recessive dystrophic epidermolysis bullosa wounds: results of a randomized, vehiclecontrolled trial. Br J Dermatol. 2013;169(5):1025-1033.

58. Caplan AI. Mesenchymal stem cells. J Orthop Res. 1991;9(5):641-650.

59. Conget P, Rodriguez F, Kramer S, et al. Replenishment of type VII collagen and re-epithelialization of chronically ulcerated skin after intradermal administration of allogeneic mesenchymal stromal cells in two patients with recessive dystrophic epidermolysis bullosa. Cytotherapy. 2010;12(3):429-431.
60. El-Darouti M, Fawzy M, Amin I, Abdel Hay R, Hegazy R, Gabr H, El Maadawi Z. Treatment of dystrophic epidermolysis bullosa with bone marrow non-hematopoeitic stem cells: a randomized controlled trial. Dermatol Ther. 2016;29(2):96-100.

61. Petrof G, Lwin SM, Martinez-Queipo M, et al. Potential of systemic allogeneic mesenchymal stromal cell therapy for children with recessive dystrophic epidermolysis bullosa. J Invest Dermatol. 2015; 135(9):2319-2321.

62. Wagner JE, Ishida-Yamamoto A, McGrath JA, et al. Bone marrow transplantation for recessive dystrophic epidermolysis bullosa. $N$ Engl J Med. 2010;363(7):629-639.

63. Geyer MB, Radhakrishnan K, Giller R, et al. Reduced toxicity conditioning and allogeneic hematopoietic progenitor cell transplantation for recessive dystrophic epidermolysis bullosa. J Pediatr. 2015; 167(3):765-9 e1.

64. TocknerB, KocherT, Hainzl S, Reichelt J, Bauer JW, KollerU, MurauerEM. Construction and validation of an RNA trans-splicing molecule suitable to repair a large number of COL7A1 mutations. Gene Ther. 2016;23(11):775-784

65. Sebastiano V, Zhen HH2, Haddad B, et al. Human COL7A1-corrected induced pluripotent stem cells for the treatment of recessive dystrophic epidermolysis bullosa. Sci Transl Med. 2014;6(264):264ra163.

66. Chen M, O’Toole EA, Muellenhoff M, Medina E, Kasahara N, Woodley DT. Development and characterization of a recombinant truncated type VII collagen "minigene". Implication for gene therapy of dystrophic epidermolysis bullosa. J Biol Chem. 2000;275(32):24429-24435.

67. Chen M, Kasahara N, Keene DR, et al. Restoration of type VII collagen expression and function in dystrophic epidermolysis bullosa. Nat Genet. 2002;32(4):670-675.

68. Osborn MJ, Starker CG, McElroy AN, et al. TALEN-based gene correction for epidermolysis bullosa. Mol Ther. 2013;21(6):1151-1159.

69. Tolar J, Xia L, Lees CJ, et al. Keratinocytes from induced pluripotent stem cells in junctional epidermolysis bullosa. J Invest Dermatol. 2013; 133(2):562-565.

70. Tolar J, Xia L, Riddle MJ, et al. Induced pluripotent stem cells from individuals with recessive dystrophic epidermolysis bullosa. J Invest Dermatol. 2011;131(4):848-856.

71. Mavilio F, Pellegrini G, Ferrari S, et al. Correction of junctional epidermolysis bullosa by transplantation of genetically modified epidermal stem cells. Nat Med. 2006;12(12):1397-1402.

72. Woodley DT, Wang X, Amir M, et al. Intravenously injected recombinant human type VII collagen homes to skin wounds and restores skin integrity of dystrophic epidermolysis bullosa. J Invest Dermatol. 2013; 133(7):1910-1913.

73. Wang X, Ghasri P, Amir M, et al. Topical application of recombinant type VII collagen incorporates into the dermal-epidermal junction and promotes wound closure. Mol Ther. 2013;21(7):1335-1344.

74. Hovnanian A. Systemic protein therapy for recessive dystrophic epidermolysis bullosa: how far are we from clinical translation? J Invest Dermatol. 2013;133(7):1719-1721.

75. Palazzi X, Marchal T, Chabanne L, Spadafora A, Magnol JP, Meneguzzi G. Inherited dystrophic epidermolysis bullosa in inbred dogs: a spontaneous animal model for somatic gene therapy. J Invest Dermatol. 2000;115(1):135-137.

76. South AP, Uitto J. Type VII collagen replacement therapy in recessive dystrophic epidermolysis bullosa-how much, how often? J Invest Dermatol. 2016;136(6):1079-1081.

77. Woodley DT, Cogan J, Wang X, et al. De novo anti-type VII collagen antibodies in patients with recessive dystrophic epidermolysis bullosa J Invest Dermatol. 2014;134(4):1138-1140.

78. Krakowski AC, Ghasri P. Case report: rapidly healing epidermolysis bullosa wound after ablative fractional resurfacing. Pediatrics. 2015; 135(1):e207-e210. 


\section{Publish your work in this journal}

Chronic Wound Care Management and Research is an international, peer reviewed, open access, online journal publishing original research, reviews, editorials, and commentaries on the causes and management of chronic wounds and the major issues related to chronic wound management. Topics also include chronic wounds as comorbidities to other conditions, patient adherence to therapy, and the economic burden of chronic wounds. The manuscript management system is completely online and includes a very quick and fair peer review system, which is all easy to use. Visit http://www.dovepress.com/testimonials.php to read real quotes from published authors.

Submit your manuscript here: https://www.dovepress.com/chronic-wound-care-management-and-research-journal 